\title{
Elaboration of diagnostic means for detection antibodies to Newcastle disease virus
}

\author{
Oksana F. Blotska* \\ Biotechnology and Control of Quality of Viral Preparations, The State Scientific-Control Institute of Biotechnology and Strains of \\ Microorganisms, Kyiv, Ukraine
}

\section{Objective}

A test kit for the detection of antibodies to Newcastle disease virus (NDV) based on haemagglutination inhibition (HI) assay has been developed and introduced into practice for the first time in Ukraine.

\section{Introduction}

Newcastle disease (ND) is the most important infectious viral disease of poultry. The world-wide economic loss from it is 2-3 billion USD per year. ND is reportable to the World Organization for Animal Health (OIE). ND is caused by virulent strains of avian Paramyxoviruses belonging to type 1 . Industrial poultry farming is rapidly developing in Ukraine. Ornithological fauna of Ukraine includes about four hundred species of birds, 207 of which nest within its borders. The territory of Ukraine transits 3 out of 14 transcontinental global migration flows. The wild birds are the main natural reservoir of ND agents. It is necessary to control the intensity of post-vaccination immunity in poultry and the timing of revaccinations. OIE recommends enzyme linked immunosorbent assays (ELISA) and HI test for these purposes [1]. However, it should be noted that HI test, possessing high specificity and sensitivity, is much cheaper. Therefore, it is the excellent means for ND timely surveillance.

\section{Methods}

During the development of a new diagnostic kit, we used the reference strain "La-Sota", which was obtained from the National Center of Microorganism Strains of Ukraine. We have produced haemagglutinating antigen using embryonated SPF fowl eggs and 10-11 day incubation. A dilution of the virus was inoculated in $0.1 \mathrm{ml}$ volumes into the allantoic cavity and incubated at $35-37^{\circ} \mathrm{C}$ for $80-96$ hours. For the purpose of NDV inactivation, we used aminoethyleneimine at the final concentration of $0.1 \%$. Positive serum was prepared by immunizing 60-day-old chickens with live virus once and by inactivated virus twice with an interval of 2 weeks. Negative serum was obtained from healthy birds that did not contain antibodies to NDV. The investigated blood sera were inactivated by heating (56 C/30 minutes). Samples of 1\% suspension of chicken erythrocytes in phosphate buffered saline (pH 7.0-7.1) were used in HI tests.

\section{Results}

The specific haemagglutination activity of the obtained antigen amounted to $10-11 \log _{2}$. The test was performed using the 4HA units of the antigen. Positive control serum activity was in the range of 7-9 $\log _{2}$. Negative control serum did not give results of more than $2 \log _{2}$.

The estimation of the quality indexes of the components of the diagnostic test-kit was performed using harmonized methods. In order to examine sensitivity and specificity of $\mathrm{HI}$ test kits, antigens and sera from commercial diagnostic kits were used. Also, certified negative control serum and samples of International Standard sera were used, which were obtained from reference laboratories, namely against the following pathogens: Avian Influenza A (H5), Avian Influenza A (H7), Egg Drop Syndrome'76 Virus, Paramyxoviruses of 2 and 3 serotypes, Reovirus, Avian Infectious Laryngotracheitis, Avian Infectious Bronchitis Virus, Mycoplasma gallisepticum, and NDV.
In order to ensure a high degree of specificity for the antigen, special attention was given to the selection of a stabilizer for freezedrying (the subject of a patent).

Comparison between the national diagnostic test kit for HI and commercial ELISA kit (IDEXX) in the evaluation of humoral immune response to ND in vaccinated chickens was investigated by examining of serum samples $(\mathrm{n}=152)$. Statistical analysis of data showed that the correlation coefficient for the results of both tests was 0.92 . The relative sensitivity of $\mathrm{HI}$ test kit was $93.5 \%$ and the relative specificity $-91.5 \%$.

The developed test kit was successfully used for the examination of field samples. We developed regulatory documents, completed the procedure of validation and registration in Ukraine of the commercial $\mathrm{HI}$ test kit for the detection of antibodies to NDV.

\section{Conclusions}

The use of the national standardized diagnostic test kit based on $\mathrm{HI}$ for detection of antibodies to NDV allows assessing the postvaccination antibodies level that helps to maintain the disease-free status of the Ukrainian poultry industry with regard to ND.

\section{Keywords}

Newcastle; disease; haemagglutination; inhibition; surveillance

\section{References}

1. OIE. Newcastle disease. In : Office International Des Epizooties Manual of standards for diagnostic tests and vaccines. Chapter 2.3.14 http://www.oie.int/ Newcastle dis.pdf.2012.

\section{*Oksana F. Blotska}

E-mail: blotskaya@ua.fm 